\title{
Efeito de diferentes concentrações de óleo e tintura de citronela sobre os parâmetros reprodutivos de teleóginas de Rhipicephalus (Boophilus) microplus
}

\section{Effect of different concentrations of citronella oil and tincture on reproductive parameters of Rhipicephalus (Boophilus) microplus teleogines}

\author{
Fernanda Carlini Cunha dos Santos ${ }^{1 *}$; Fernanda Silveira Flores Vogel ${ }^{2}$; \\ Silvia Gonzalez Monteiro ${ }^{3}$
}

\section{Resumo}

O carrapato Rhipicephalus (Boophilus) microplus é o ectoparasito mais importante em áreas de exploração pecuária, sendo responsável por severas perdas econômicas. O controle deste parasito é feito através de produtos químicos, que são utilizados de forma indiscriminada e resultam na redução da eficiência destes compostos. A fim de retardar o aparecimento da resistência parasitária, alguns métodos de controle alternativo vêm sendo pesquisados, entre eles a fitoterapia. O objetivo deste experimento foi avaliar a eficiência in vitro do óleo e da tintura de citronela (Cymbopogon nardus) sobre fêmeas ingurgitadas de $R$. (B) microplus seguindo a metodologia do exame de biocarrapaticidograma. Para isto foram testadas 4 diluições do óleo $(1 ; 25 ; 50$ e 100\%), sendo que a inibição de postura foi de $16 ; 40 ; 53$ e $73 \%$, a eclosão larval de $28 ; 16 ; 23$ e $6 \%$ e a eficácia de $78 ; 100 ; 86$ e $98 \%$, respectivamente. O gradativo aumento da inibição da postura e a baixa eclosão larval influenciaram positivamente na eficiência dos tratamentos. A solução do óleo a $1 \%$ apresentou controle parcial das teleóginas e a solução a $25 \%$ apresentou $100 \%$ de eficiência. Foram testadas 6 diluições da tintura de citronela $(1 ; 5 ; 10 ; 25 ; 50$ e $100 \%$ ), sendo que a inibição da postura foi de $10 ; 63 ; 80 ; 83 ; 86$ e $96 \%$, a eclosão larval de $91 ; 31$; 6; 26; 0 e $0 \%$ e a eficácia de 23; 93; 94; 97; 100 e 100\%, respectivamente. A maioria dos tratamentos com a tintura de citronela apresentou inibição de postura superior a $80 \%$ e baixa taxa de eclosão larval. A tintura a 5\% teve eficácia semelhante aos tratamentos com maiores concentrações. Na avaliação estatística foi verificado associação significativa entre a concentração do óleo e da tintura de citronela com a eficiência do tratamento. Conclui-se que tanto o óleo como a tintura de $C$. nardus demonstraram efeito negativo nos parâmetros reprodutivos do carrapato $R$. (B) microplus, em experimento in vitro. Palavras-chave: Acaricida, agroecologia, Cymbopogon nardus, ectoparasitas, fitoterapia, plantas medicinais

\footnotetext{
Abstract

The tick Rhipicephalus (Boophilus) microplus is the most important ectoparasite in cattle rising, and it is responsible for severe economic losses. Parasite control is based on chemicals, which are used indiscriminately and result in effectiveness reduction of these compounds. In order to delay the onset

${ }^{1}$ Médica Veterinária Autônoma. Universidade Federal de Santa Maria, RS. E-mail: carlini@portoweb.com.br

${ }^{2}$ Prof $^{a}$ Dr $^{\mathrm{a}}$ do Dept ${ }^{\mathrm{o}}$ de Medicina Veterinária Preventiva, Laboratório de Doenças Parasitárias, Universidade Federal de Santa Maria, UFSM, Santa Maria, RS. E-mail: fefvogel@gmail.com

${ }^{3}$ Prof $^{\mathrm{a}} \mathrm{Dr}^{\mathrm{a}}$ do Dept $^{\mathrm{o}}$ de Microbiologia e Parasitologia, Laboratório de Parasitologia Veterinária. UFM, Santa Maria, RS. E-mail:
} sgmonteiro@uol.com

* Autor para correspondência 
of parasite resistance, some alternative methods are being researched, including herbal medicine. The aim of this experiment was to evaluate the in vitro efficacy of citronella (Cymbopogon nardus) oil and tincture on $R$. (B) microplus by the engorged female immersion test. Four oil concentrations were tested $(1 ; 25 ; 50 ; 100 \%)$ and the inhibition of oviposition were $16 ; 40 ; 53 ; 73 \%$, egg hatch were $28 ; 16 ; 23$; $6 \%$ and the effectiveness were $78 ; 100 ; 86 ; 98 \%$, respectively. The gradual rise of the inhibition of oviposition and low egg hatch had a positive influence in the effectiveness of treatment. The solution at $1 \%$ of citronella oil had a partial control of the teleogines and the solution at $25 \%$ had $100 \%$ of effectiveness. Six dilutions of the tincture $(1 ; 5 ; 10 ; 25 ; 50 ; 100 \%)$ were tested and the inhibition of oviposition were $10 ; 63 ; 80 ; 83 ; 86 ; 96 \%$, egg hatch were $91 ; 31 ; 6 ; 26 ; 0 ; 0 \%$ and the effectiveness were $23 ; 93 ; 94 ; 97 ; 100 ; 100 \%$, respectively. Most of the treatments with the citronella tincture had inhibition of oviposition higher than $80 \%$ and low egg hatch rate. The tincture at $5 \%$ had similar efficacy compared to the groups with higher concentrations. A significant association between the oil and tincture of citronella and the treatment effectiveness was observed in the statistical evaluation. It is possible to conclude that both the oil and the tincture of citronella had negative influence in the reproductive parameters of $R$. (B) microplus.

Key words: Acaricide, agribusiness, Cymbopogon nardus, ectoparasites, medicinal plants, phytoterapic

O método de controle do carrapato Rhipicephalus (Boophilus) microplus mais preconizado é a aplicação de produtos químicos e um dos fatores mais preocupantes é o surgimento e estabelecimento de resistência parasitária aos produtos empregados. Outro severo problema é a contaminação do meio ambiente com o uso excessivo de carrapaticidas químicos, ocasionando a contaminação do solo, dos recursos hídricos e promovendo transtornos à saúde coletiva (PEREIRA; FAMADAS, 2004).

Por este motivo, pesquisadores das diferentes regiões do mundo têm buscado alternativas visando diminuir o uso de acaricidas sintéticos (JONSSON; DAVIS; WITT, 1998). Uma destas alternativas é o uso de plantas medicinais, devido principalmente à grande variabilidade de espécies existentes, baixo custo, fácil disponibilidade, rápida degradação, ausência de contaminação do ambiente e, consequentemente, dos animais e do homem (AGNOLIN, 2009).

Produtos a base de citronela são utilizados como repelentes, inseticidas de moscas e mosquitos (RAJA et al., 2001) e diversas pesquisas vêm sendo desenvolvidas acerca de sua ação carrapaticida. A composição química de um fitoterápico é complexa e pode ser avaliada através de análise cromatográfica. As principais propriedades da citronela são atribuídas à substâncias voláteis em suas folhas, como citronelal, eugenol, geramiol, elemol e limoneno, entre outras, denominadas de monoterpenos (SHASANY et al., 2000). O rendimento do óleo essencial de citronela com base na matéria in natura é de aproximadamente 0,5\% e os principais métodos de extração tem como base o uso de vapor, álcool ou material pré-secado. A utilização da citronela de forma isolada ou combinada com princípios ativos sintéticos pode-se constituir uma alternativa viável e mais ecológica no combate do carrapato bovino.

Este experimento teve como objetivo avaliar da eficiência de diferentes concentrações do óleo e da tintura de citronela (Cymbopogon nardus) sobre fêmeas ingurgitadas de $R$. (B) microplus, em ensaio in vitro.

Os testes foram conduzidos no Laboratório de Doenças Parasitárias da Universidade Federal de Santa Maria, Rio Grande do Sul (RS), entre os meses de dezembro de 2008 e abril de 2009. Foram colhidas teleóginas de $R$. (B) microplus, em bovinos naturalmente infestados de uma propriedade de Santa Maria, sendo que no último tratamento foi administrado ivermectina ${ }^{4}$ há aproximadamente 70 dias antes da coleta. No laboratório, as mesmas foram lavadas em água corrente, secas em papel toalha e separadas em 10 tratamentos, com 10

4 Ivomec Injevátel 1\%, Merial, Campinas, SP. 
teleóginas cada, seguindo a técnica descrita por Drummond et al. (1973). O óleo e a tintura de citronela foram adquiridos da empresa Tecpon (Cachoeirinha, RS), sendo que este é extraído da planta através do método de arraste de vapor.

O tratamento I foi considerado controle não tratado e neste utilizou-se água destilada. Para o óleo de citronela utilizou-se nos tratamentos II a $\mathrm{V}$ as concentrações de 1, 25, 50 e 100\%, respectivamente, diluídos em água destilada. Para a tintura de citronela utilizou-se nos tratamentos II a VII as concentrações 1, 5, 10, 25, 50 e 100\%, respectivamente, diluídos em água destilada. As teleóginas foram imersas em $20 \mathrm{~mL}$ das soluções correspondentes a cada tratamento por 5 minutos e agitadas manualmente a cada 30 segundos. Todos os tratamentos foram realizados em triplicata e os resultados foram obtidos através de suas médias. Após a imersão, as teleóginas foram secas em papel toalha e fixadas através de uma fita adesiva em placas de Petri, previamente identificadas. A seguir foram levadas a estufa climatizada, regulada a temperatura de $27{ }^{\circ} \mathrm{C}$ e umidade relativa superior a $80 \%$, por 14 dias. Após o período de ovipostura, esta foi pesada em alíquotas de $0,3 \mathrm{~g}$ de ovos e armazenadas em tubos de ensaio, vedados com algodão hidrófobo, sendo, novamente encaminhados para a estufa, onde foram mantidos por 26 dias. Após o período de incubação, realizou-se a leitura do percentual de eclodibilidade. A eficiência do tratamento foi determinada a partir do cálculo do índice de inibição da reprodução, segundo Drummond et al. (1973), e a avaliação estatística foi realizada através de análise de regressão, a um nível de significância de 5\%.

Conforme observado na Tabela 1, o tratamento com $1 \%$ do óleo de citronela exerceu ação negativa sobre as teleóginas, pois inibiu parte da postura e da eclosão larval. A inibição da postura foi inversamente proporcional à concentração, no entanto, esta postura foi inibida somente nos grupos com as maiores concentrações do óleo. A eclosão das larvas foi baixa em todos os tratamentos $(6,23 \mathrm{e}$ $28 \%$ ). Apesar da solução a $50 \%$ ter sido superior na inibição da postura, a eclosão larval foi similar com o tratamento a $1 \%$. O tratamento com $100 \%$ obteve eficácia similar, porém inferior à concentração de $25 \%$. O tratamento com $25 \%$ do óleo obteve eficácia máxima. Sugere-se mais pesquisas a cerca das soluções entre $1 \%$ a $25 \%$ do óleo, sendo estas mais economicamente viáveis e com alta eficiência.

Tabela 1. Médias percentuais (\%) de inibição de postura (IP), eclosão larval (EL), e eficiência do tratamento (ET) das diferentes concentrações do óleo de Cymbopogon nardus (C) sobre teleóginas de Rhipicephalus (Boophilus) microplus.

\begin{tabular}{ccccc}
\hline TRATAMENTO & C & IP & EL & ET \\
\hline I & 0 & 0 & 100 & 0 \\
II & 1 & 16 & 28 & 78 \\
III & 25 & 60 & 0 & 100 \\
IV & 50 & 53 & 23 & 86 \\
V & 100 & 73 & 6 & 98 \\
\hline
\end{tabular}

Fonte: Elaboração dos autores.

Conforme exposto na Tabela 2, a elevada eficácia da tintura de citronela foi devido à alta inibição da postura e baixa eclosão larval. Nesta pesquisa, a partir de 5\% de concentração da tintura, os resultados de eficácia foram similares. Os tratamentos com 10 , 25 e $50 \%$ da tintura tiveram inibição de postura semelhante ( 80,83 e $86 \%$ ), consequentemente, com eficácia similar, no entanto com diferentes taxas de eclosão larval $(6,26$ e 0\%). As concentrações de 50 e $100 \%$ tiveram eficácia máxima, porém estas soluções são inviáveis para uso in vivo, pelo valor econômico. 
Tabela 2. Médias percentuais (\%) de inibição de postura (IP), eclosão larval (EL), e eficiência do tratamento (ET) das diferentes concentrações da tintura de Cymbopogon nardus (C) sobre teleóginas de Rhipicephalus (Boophilus) microplus.

\begin{tabular}{ccccc}
\hline TRATAMENTO & C & IP & EL & ET \\
\hline I & 0 & 0 & 100 & 0 \\
II & 1 & 10 & 91 & 23 \\
III & 5 & 63 & 31 & 93 \\
IV & 10 & 80 & 6 & 94 \\
V & 25 & 83 & 26 & 97 \\
VI & 50 & 86 & 0 & 100 \\
VII & 100 & 96 & 0 & 100 \\
\hline
\end{tabular}

Fonte: Elaboração dos autores.

Em relação às variáveis inibição de postura $(x)$ e concentração do óleo de citronela (y) foi encontrada na análise de regressão linear diferença significativa $(\mathrm{P}=0,007)$ entre as diferentes soluções $(\mathrm{y}=21,0$ $+0,614 x)$. Para eclosão larval $(x)$ e concentração do óleo (y) foi verificada associação significativa $(y=55,1-0,585, P=0,027)$. Para eficiência do tratamento $(\mathrm{x})$ e concentração da citronela (y) também foi verificado associação significativa $(\mathrm{P}$ $=0,031)$ expressa na equação $\mathrm{y}=52,5+0,572 \mathrm{x}$ (Gráfico 1). Na análise de regressão linear com os dados de inibição de postura (x) e concentração da tintura de citronela (y) foi verificado associação significativa $(\mathrm{P}=0,002)$ expressa na equação $\mathrm{y}=$ $39,7+0,726 x$. Em relação a eclosão de postura $(x)$ e concentração da tintura $(\mathrm{y})$ foi verificado associação significativa $(\mathrm{P}=0,005)$ expressa na equação y $=57,1-0,749 x$. Com os dados de eficácia do tratamento $(\mathrm{x})$ e concentração da tintura (y) foi verificado associação significativa $(\mathrm{P}=0,012)$ expressa na equação $\mathrm{y}=55,7+0,620 \mathrm{x}$ (Gráfico 2). Estes cálculos indicam que a concentração da citronela, tanto na forma de tintura como de óleo, apresenta um aumento proporcional com a inibição de postura, inversamente com a eclosão larval e proporcional com a eficiência do tratamento.
Agnolin (2009) em seu experimento in vivo constatou que houve declínio no número de teleóginas a partir do $14^{\circ}$ dia da aplicação do óleo de citronela a $3 \%$. No tratamento com $4 \%$ isso ocorreu a partir do $3^{\circ}$ dia. Estes resultados corroboram com os do presente trabalho, onde o aumento da ação acaricida foi proporcional ao aumento da concentração. Esta ação deve-se provavelmente ao princípio ativo citronelal, componente de maior participação no óleo e de comprovada ação inseticida e acaricida (CHAGAS et al., 2002).

Vendrame et al. (2007), em seus experimentos in vivo, observaram que a concentração de $6 \%$ de citronela apresentou melhor desempenho, mantendo até o $7^{\circ}$ dia pós-tratamento um nível de infestação inferior ao observado no pré-tratamento. No presente estudo, a tintura a $5 \%$ apresentou eficiência de $93 \%$, classificada como de média eficácia. Este mesmo pesquisador constatou que o tratamento constituído por $12 \%$ de citronela teve eficácia semelhante ao grupo controle. Porém, no atual trabalho, a tintura a $10 \%$ teve eficácia semelhante a do tratamento com $5 \%$, discordando de Vendrame et al. (2007), apesar das diferenças na metodologia. 
Gráfico 1. Análise de regressão linear com os parâmetros de eficiência do tratamento (ET) (x) e concentração do óleo de Cymbopogon nardus (\%) (y) em teleóginas de Rhipicephalus (Boophilus) microplus, Santa Maria, RS.

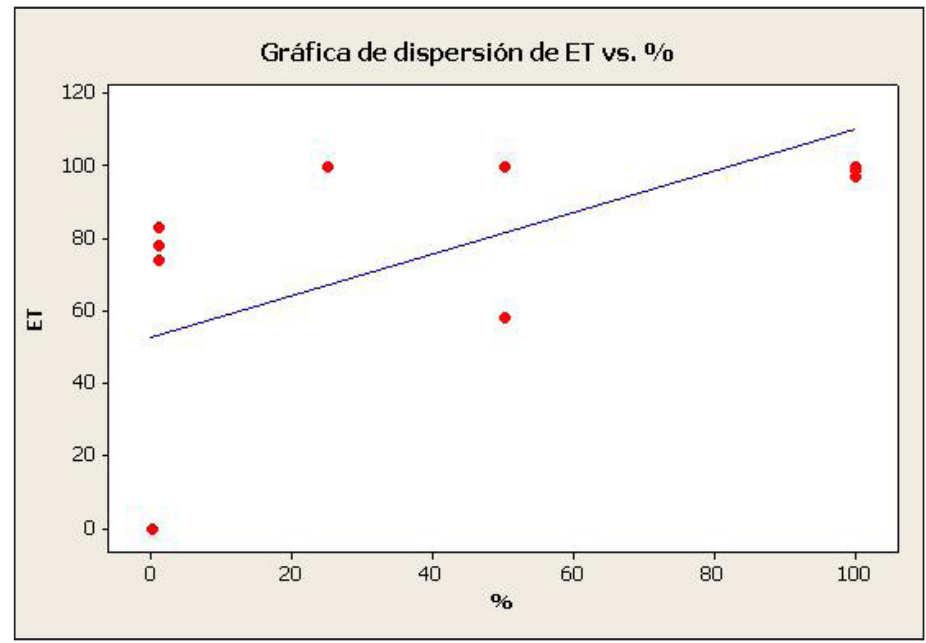

Fonte: Elaboração dos autores.

Gráfico 2. Análise de regressão linear com os parâmetros de eficiência do tratamento (ET) (x) e concentração da tintura de Cymbopogon nardus (\%) (y) em teleóginas de Rhipicephalus (Boophilus) microplus, Santa Maria, RS.



Fonte: Elaboração dos autores.

A ação acaricida deste fitoterápico foi e de $100,89,86$ e $66 \%$ no $5^{\circ}$ dia pós-tratamento. comprovada por Chungsamarnyart e Jiwajinda (1992) que utilizando o óleo de citronela a 12,5; Estes resultados in vivo são semelhantes ao que foi 8,3 e 7,1\% verificaram uma ação larvicida de 95,7; encontrado em nossos testes in vitro. No laboratório 92,7 e $58,1 \%$, respectivamente. Os mesmos autores usando formulações constituídas por 100, 75, 50 e $25 \%$ de óleo de citronela em etanol, aplicadas sob a forma de aspersão sobre animais naturalmente infestados, observaram mortalidade de 100, 70, 70 e $24 \%$ das teleóginas 48 horas após o tratamento observou-se maior mortalidade de teleóginas em soluções com menores concentrações de citronela. Estes autores conseguiram grande ação larvicida com concentrações inferiores às testadas neste experimento, onde o tratamento com $1 \%$ do óleo causou redução de $62 \%$ na emergência de larvas, possivelmente devido a diferenças na formulação do produto. 
Em estudos relacionados com outra espécie de Cymbopogon, o óleo de citronela de Java (Cymbopogon winterianus), foi constatado uma eficácia de $50 \%$ sobre teleóginas e larvas, usando concentrações de $6,1 \%$ e $4,1 \%$, respectivamente (MARTINS, 2006). Também foi verificado que não houve postura quando as teleóginas foram imersas no óleo a $10 \%$ e tão pouco ocorreu eclosão larval na concentração de 7,14\%. Segundo o mesmo autor, a citronela de Java é constituída de citronelal e geraniol, que quando estudados isoladamente, demonstraram efeito acaricida diferentes. O geraniol tem poder acaricida superior ao citronelal, no entanto, o autor sugere que estes dois componentes possuem ação sinérgica sobre o carrapato.

Olivo et al. (2008) testando várias concentrações do óleo de citronela $(0,1 ; 0,25 ; 0,5 ; 1 ; 2 ; 10 ; 25$ e 50) encontraram eficácia de 0,$7 ; 2,8 ; 51,6 ; 79,3$; $81 ; 87,1 ; 86,7$ e $89,5 \%$, respectivamente. Estes resultados são na maioria similares aos deste experimento, concordando com ação do óleo de citronela in vitro sobre o carrapato bovino.

Gularte et al. (2009) encontraram média de eficácia de 80; 94 e 100\% para as concentrações de 6,$25 ; 12,5$ e $25 \%$ de óleo de citronela, corroborando com os resultados do presente experimento. Estes autores constataram que a mortalidade das teleóginas foi crescente e a eclodibilidade foi decrescente de acordo com o aumento da concentração. A eficácia da solução a $25 \%$ foi a mesma do presente experimento, onde também foi constatado o mesmo efeito em relação ao aumento da concentração.

Os resultados obtidos no presente experimento demonstram um efeito negativo da citronela frente à teleóginas ingurgitadas do carrapato $R$. (B) microplus. Este efeito pode atribuído a diversos fatores como composição da planta no momento da coleta, método de extração da planta, a própria formulação da solução, entre diversas outras variáveis não estudadas. Em relação à planta é interessante destacar-se que a composição do fitoterápico Cymbopogon varia conforme a família utilizada, assim como o estado da planta no momento da extração. Segundo Reis et al. (2006) para plantas em estado fresco, a temperatura adequada do ar de secagem influencia na qualidade e rendimento durante a extração o óleo essencial, constatando também que a planta em estado fresco apresenta menor perda dos compostos voláteis em comparação as que foram secas e/ou congeladas.

Os resultados são bastante promissores em relação ao uso do óleo e da tintura de citronela no controle do carrapato bovino. O tratamento com $1 \%$ do óleo de citronela apresentou controle parcial sobre as teleóginas, e a partir de $25 \%$ teve eficácia máxima. O tratamento com $1 \%$ da tintura de citronela teve baixa eficiência. A partir de 5\% da tintura todos os grupos tiveram eficácia superior a 90\%. O óleo e a tintura de citronela apresentaram ação negativa sobre os parâmetros reprodutivos do carrapato $R$. (B) microplus em experimento in vitro. Formulações contendo óleo ou tintura de citronela podem ser uma interesse alternativa para uso no controle do carrapato, sendo seu uso de forma isolada e em maior concentração ou de forma combinada com produtos químicos e em menor concentração. Sugere-se que mais pesquisas sejam realizadas com estes dois enfoques, a fim de reduzir a contaminação ambiental, frequência de tratamentos e desacelerar a seleção de cepas com resistência parasitária múltipla.

\section{Referências}

AGNOLIN, C. A. Óleo de citronela no controle de ectoparasitas de bovinos. 2009. Tese (Mestrado em Produção Animal/Bovinocultura Leiteira) - Universidade Federal de Santa Maria, Santa Maria.

CHAGAS, A. C. S.; PASSOS, W. M.; PRATES, H. T.; LEITE, R. C.; FURLONG, J.; FORTES, I. C. P. Efeito acaricida de óleos essenciais e concentrados emulsionáveis de Eucalyptus spp em Boophilus microplus. Brazilian Journal of Veterinary Research and Animal Science, São Paulo, v. 39, n. 5, p. 247-253, 2002. 
CHUNGSAMARNYART, N.; JIWAJINDA, S. Acaricidal activity of volatile oil from lemon and citronella grasses on tropical cattle ticks. Kasetsart Journal, Bankcock, v. 26, n. 5, p. 46-51, 1992.

DRUMMOND, R. O.; ERNST, S. E.; TREVINO, J. L.; GLADNEY, W. J.; GRAHAM, O. H. Boophilus annulatus and Boophilus microplus: laboratory tests for insecticides. Journal of Economic Entomology, Laham, v. 66, n. 1, p. 130-133, 1973.

GUlarte, V. N.; CASTRO, N. A.; LOPES, A.; GOUVÊA, D.; NEVES, A. M.; FREITAG, R.; SILVA, S. S.; NIZOLI, L.; SANTOS, T. R. B. Análise comparativa da eficácia de acaricidas comerciais e fitoterápicos pelo teste de Drummond et al. (1973) em Rhipicephalus (B.) microplus. In: CIC XI ENPOS, 18; AMOSTRA CIENTÍFICA DE DA UFPEL, 1., 2009, Pelotas. Anais... Pelotas: Gráfica Universitária da UFPEL, 2009. 51 p.

JONSSON, N. N.; DAVIS, R.; WITT, M. Production effects of cattle tick (Boophilus microplus) infestation of high yielding dairy cows. Veterinary Parasitology, Amsterdam, v. 78, n. 1, p. 65-77, jul. 1998.

MARTINS, R. M. Estudo in vitro da ação acaricida do óleo essencial da gramínea Citronela de Java (Cymbopogon winterianus Jowitt) no carrapato Boophilus microplus. Revista Brasileira de Plantas Medicinais, Botucatu, v. 8, n. 2, p. 71-78, 2006.

OliVO, C. J.; CARVALHO, N. M.; SILVA, J. H. S.; VOGEL, F. F.; MASSARIOL, P.; MEINERZ, G.; AGNOLIN, C.; MOREL, A. F.; VIAU, L.V. Óleo de citronela no controle do carrapato de bovinos. Ciência Rural, Santa Maria, v. 38, n. 2, p. 406-410, 2008.
PEREIRA, J. R.; FAMADAS, K. M. Avaliação "in vitro" do extrato da raiz do timbó (Dahlstedtia pentaphylla) (Leguminosae, Papilionoidae, Millettiedae) sobre Boophilus microplus (Canestrini, 1887) na região do Vale do Paraíba, São Paulo, Brasil. Arquivo do Instituto Biológico, São Paulo, v. 71, n. 4, p. 443-450, 2004.

RAJA, N.; ALBERT, S.; IGNACIMUTHU, S.; DORN, $\mathrm{S}$. Effect of volatile oils in protecting stored Vigna unguiculata (L.) Walpers against Callosobruchus maculatus (F.) (Coleóptera: Bruchidae) infestation. Journal of Stored Products Research, Denver, v. 37, n. 2, p. 127-132, 2001.

REIS, G. G.; PEISINO, A.; ALBERTO, D. L.; MENDES, M. F.; CALÇADA, L. A. Estudo do efeito da secagem em convecção naturaç e forçada na comporsição do óleo essencial de citronela (Cymbopogon nardus). Revista Brasileira de Plantas Medicinais, Botucatu, v. 8, n. 4, p. 47-55, 2006.

SHASANY, A. K.; LAU, L. K.; PATRA, N. K.; DAROKAR, M. P.; GARG, A.; KUMAR, S.; KHANUJA, S. P. S. Phenotypic and RAPD diversity among Cymbopogon Winterianus Jowitt accessions in relation to Cymbopogon nardus Rendle. Genetic Resources and Crop Evolution, Holanda, v. 47, n. 5, p. 553-559, 2000.

VENDRAME, R.; OLIVO, C. J.; MEINERZ, G. R.; AGNOLIN, C. A.; ZIECH, M. F.; HOHENREUTHER, F.; DIEHL, M.; STEINWANDTER, E. Extrato aquoso de citronela no controle do carrapato de bovinos. Cadernos de Agroecologia, Porto Alegre, v. 2, n. 2, p. 1544-1547, 2007. 
